\title{
SLA application with Big Data on WSQ Taxonomy
}

\author{
Asiya Abdus Salam \\ University of Dammam \\ Dammam, KSA
}

\author{
Reem Alzoubi \\ University of Hail \\ Hail, KSA
}

\begin{abstract}
Web taxonomy is used for better web searches. Web Supported Query taxonomy classifier produces better searching result. In order to achieve this goal with more advance methodology, big data technology with Service Level Agreement (SLA) is combined and discussed as a paradigm in this paper. The queries are directed to the cloud categorization of big data for improved result using SLA matching that enables automatic selection of most favorable services. Big data, which is a collection of huge data, enhance the performance of web searching in a united pool of resources. For the regularity of controlled vocabularies and immediacy and relevancy of collaborative tagging, it provides a suitable method for managing the flow of information over the network

This paper is organized as follows: In the first part, SLA and Big Data is introduced. Associated work of SLA and big data is discussed in section 2. WSQ methodology and later SLA structure is defined in later sections. The paper is ended with the discussion on proposed model with the conclusion and future direction.
\end{abstract}

\section{Keywords}

Service Level Agreement, SLA, Big Data, Cloud computing, Web supported query taxonomies, SLA matching and SLA management.

\section{INTRODUCTION}

People's perception is transformed from the advent of cloud computing. Growth of data is needed to be handle cloud computing is used to deploy big data.

For the provision of resources, SLA grant managed services agreement or facilities management agreement so that business objective can be achieved. SLA refers to those services provided by the service provider and defined the standard or level required for those services.

As more people, equipment and networks are in connection, data is generating continuously which creates the need of data management. Discussion here begin with SLA and then later with the latest advent of big data technology.

\subsection{SLA}

SLA refers to the agreement among service provided by service provider and end user's expectations based on the definition of the standard or level required for those services. SLA defines what is received by end user. [1]

SLA provides description of service being provided that is reliable. Responsive and contain procedure for reporting. It monitors performance of data and also includes consequences for not meeting service obligations.

SLA includes details related to the service deliverables and performance standards for fulfilling the provision of services. SLA is responsible for what services are provided, where and to who provided and what is the need of it. [2]
SLA is also defined as an agreement for providing expected consumers service quality. It helped to provide service computing situation.

A Service Level Agreement (SLA) is a bond between a complex provision and a client that stipulates, usually in determinate terms, what services the network service provider will deliver and what drawbacks will consider if the service provider cannot meet the conventional objectives.

Facility suppliers' discrepancy will be focused by the consistency of the SLA Management and its observation during utilization to subsidizing the customers' expectation. For the assurance of the proper level of performance to the customers, service providers often suggest services with SLAs, which deliver customers with dimensions of measurements like network convenience, output, and expectancy.

Following is the requirement of SLA when expectation of clients is considered for providing services:

- Dimension in terms of reliability of the quality of services.

- Delivery of the normal quality of services.

Optimization of the supply of traditional SLA Management will be a main performer in the agreement of the Next Generation Services offered by the Next Generation Networks.

SLA Management will administer the self-reliance customer can have on their consumption, and support the change from old-style usage of the services, to more particularized usage of these new services.

Here, the latest advent of big data technology is under consideration. As more people, equipment and networks are connected, data is produced continuously.

\subsection{Big Data}

The term big data is defined as the capability to deal with the huge amount of data producing every second over the digital medium. Nowadays everything like activity data, conversation data, photo or video image data, sensor data etc is stored and tracked it with high speed of transfer and movement.[3]

Ever since the development of intelligence, human beings collected and processed information on an increasing scale. The advent of computers in the 20th century and their subsequent evolution to become universal, it generated a massive growth of digital data and the continuous demand for processing it.

When the size of data accumulated in an organization, it becomes huge, much beyond the scope of getting processed by conventional infrastructure prevailing there, alternate methods were thought which ended up in Big data Analysis and the associated schemes. 
The challenges poised in Big Data manipulation include collection, allocation, storage, search, sharing, analytical processing, derivative mapping and visualization. It has become an important strategy for enterprise infrastructure managers to consider Big Data capability too.

There are several popular tools and practices in the argument which can help to realize the analysis of Terra or petabytes of data for the purpose of mining or classification or deriving threads of prospective trends which are normally invisible. Visualization plays a vital role in comprehension and the domain of big data support it in many ways.

Now the question for this paper is that how big data is merged with SLA to form better result over WSQ methodology. Big data enable to find different and enhanced results. Big data can make it easy to link the data with service provider data analysis to search queries on internet.

\section{ASSOCIATED WORK}

The awareness of an SLA can be found back to 1980s especially in telecommunication sectors. SLA was actually emphasized with the arrival of Grid computing typically for commercial service oriented when users stipulate different levels of services based on SLA required for treating the task.

Monitoring also done by Sahai et. al. in 2003 on SLA, which came across some challenges in SLA management. These challenges were addressed by WSLA in 2003 and WS Agreement in 2007. Cloud management can support dynamic environment to deal SLA management. [4]

In 2004, Dinesh et al worked on SLA and came up with the conclusion that SLA is an unambiguous testimonial of opportunities and regulation that exist in a business relationship between organization service provider and customer.

Many users start using it as a service based on data centers so that they can get these services on their personal computers. Service providers fulfill the request from users and are valued by feedback mechanism. Users are encouraged and discouraged to request services.

In fulfilling the request of computing resources, utility models are used. Only few requests are accomplished out of many. Other parameters are considered for focusing the service request. Those parameters are considered reliable and trust worthy and secured factors. As there is a need of continuous checking the changes, these parameters are highly beneficial. Importance is given to users as they access the services.

Specific work in the field of SLA for web service is also found in terms of Web service agreement and Web Service Level Agreement language and framework.[5]

The history shows the need of big data as trading activity and recording inventory and also for accessing and analyzing vast data that reveals story about big data as a growth in the volume of data previously known as "information explosion". For handling too much data, actual work started on it in the era of 1944 and onward showing improvement in the quantitative as well as qualitative part of accessible and relevant data which was result of updated and enhancement in data storage technology.
In 2005 , the birth of web 2.0 related user generated content was defined. In 2008, nature on big data defines big data theories and the research continued. It is considered as one of the huge innovation in last few couples of years. The procedure includes collection, organization and processing in all fields.

In 2010, Kenneth publishes a report on big data indicating its importance showing unimaginable huge digital data spreading rapidly. Its effect is everywhere in all fields of life. In 2012, big data was defined in terms of technology, analysis and mythology. The progress continues and in 2015 , huge amount of data exploded in the history.

\section{WSQ METHODOLOGY}

Previously web supported query taxonomy works on online categorization with classifiers.[6] Here the approach proposed is based on applying category cloud with SLA matching and selection technique. It is further integrated with Web support query taxonomy for creating better results. The query is inserted from the user's end and with the help of cloud computing on demand, computing resources requests are fulfilled.

Lately, Cloud computing has appeared as a novel policy for conveying effective computing facilities. In Clouds setup, policy and request facilities are accessible on-demand and companies are capable of accessing their commercial facilities and applications everyplace in the world whenever it's essential. In this background, enormously accessible systems are made obtainable to end users as a provision.

Moreover, this picture, where both on demand rate and resources accessibility continuously vary, SLAs are used to guarantee that service superiority is kept at adequate levels despite such dynamically. As shown in Figure 1, searching and identification of resources are defined using SLA in the cloud categories.

Integration of SLA helped out in better results. Benefits like giving better satisfaction level to customers with improved quality of service and relationship between two parties are provided by SLA.

SLA will make sure the definite criteria of accessibility and in term of functionality. If there is any inactivity or network interruption or postponement when passing information from one point to other, SLA services are more required. It will alert about delays in functionality or if service becomes unavailable.

As specified in this proposed paper, it will be perceived a very genuine requirement for a vibrant and official procedure to handle SLAs in the framework of cloud computing. SLA with big data recommends a very springy architecture for handling and management of SLAs between sources and clients. In applying SLA however, the need for WSQ services arises.

One of the main points of SLA where, true to the paradigm of Service Oriented Architecture, whole functionality is delivered as a service that may not essentially come from the same source. One significant statement we make in the framework of clouds is the absence of regularization especially essential when effort is applied on observing across various clouds. [7] 


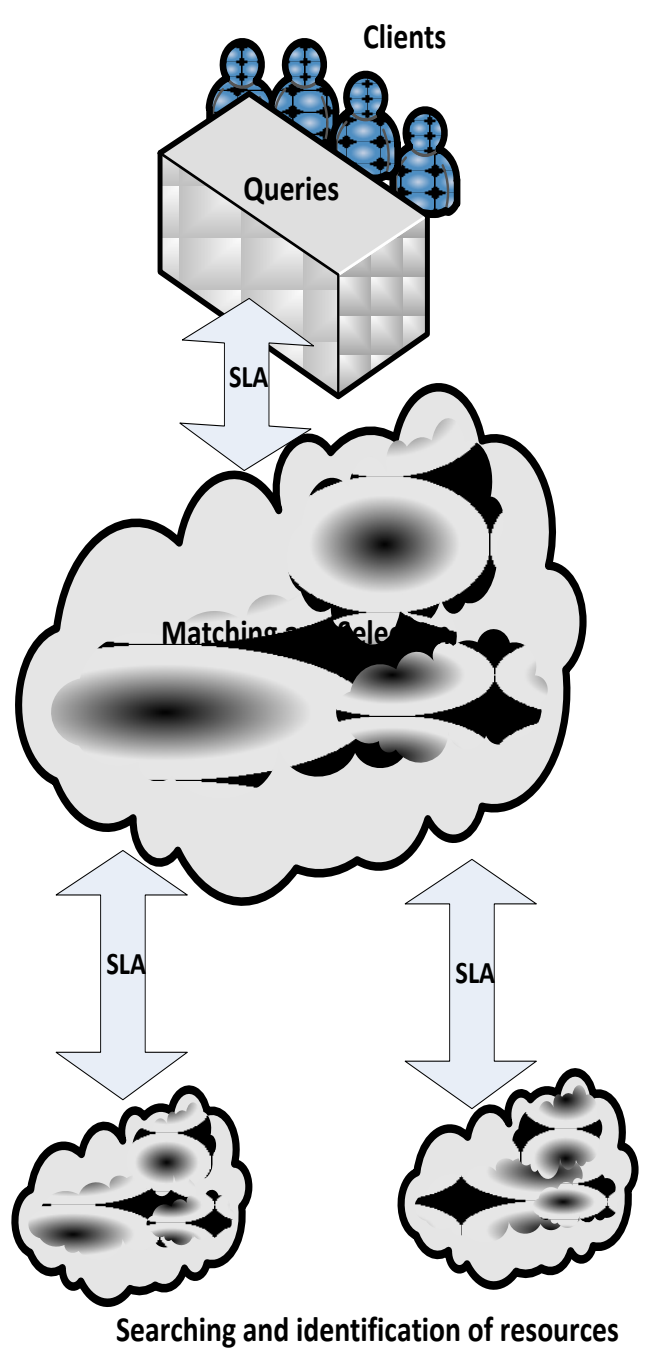

Figure 1: SLA and Big Data Clouds

\section{SLA STRUCTURE}

The SLA is a service agreement that has a contract between service provider and internal or external customer to enhance the service level and performance. The satisfied customer according to quality of services provided is a critical point because the success or fail the SLA application depend on them. Moreover most layers SLA includes both general and technical specifications, including business parties, pricing policy, and properties of the resources required to process the service as customer satisfaction.

So it needs to build the vigorous structure of SLA which described the procedure step by step that it will be followed to meet the service in optimal satisfaction. The structure of SLA is shown in Figure 2 Structure of SLA.

SLA is a powerful communication tool as it improves communication between facility providers and customers. Also, it prevents the disputes and issues and defines responsibilities. It appears as a mechanism to receive and act on complaints for the management and performed effective expectation managing tool helping in not just resolving conflicts but consider request as expected.

Therefore, SLA establishes service performance matrix for quality relationships. High quality standards and requests control services by having good method for managed system.

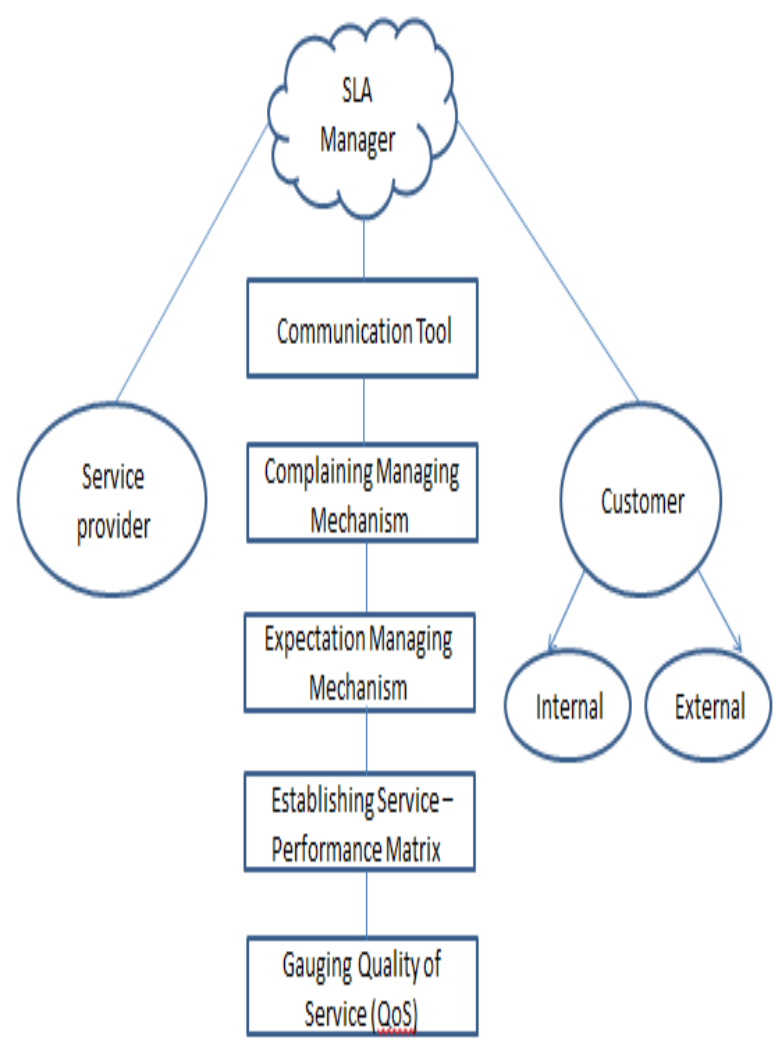

Figure 2: Structure of SLA

\subsection{How SLA works with Big Data?}

Big data is the name that describes the extreme volume of data, the wide variety of data types and the velocity at which the data must be processed. It is always characterized by $3 \mathrm{Vs}$. $3 \mathrm{Vs}$ (volume, variety and velocity) are three essential properties. Volume refers to the size of data, variety refers to the types of data and velocity refers to the speed of data processing. So it needs to manage big data in efficient way when the customer's request for any service that is called SLA application [8].

SLA manager is a responsible person who has established the SLA and controlled the big data that he/she had in his/her company. SLA needs a face to face negotiation process to set up the agreement between different parties. SLA manager must have high quality skills [9]:

- Be aware of the organization unit they present.

- Be knowledgeable about the establishment and management of SLA depending on the experiences that have.

- Be able to save the time and efforts needed to set up and manage the agreement.

- $\quad$ Be skilled to seek win -win relationship.

- Be friendly, personally respected and other part's organization.

Once an SLA becomes operational, the SLA manager has responsibilities to make successful the SLA application [9]:

- Serving as the point of communication for problems or worries related to the SLA itself and the delivery of services described in the SLA. 
- Organizing and implementing changes on service delivery and on the SLA itself.

- Planning and coordinating of the services that requested by customer.

- Regularly assessing and reporting customers complains to prevent them in future requirements.

- Conducting any issues that improve the strength and quality of service.

It needs to follow sequential steps for achieved optimal results. The first step for establishing SLA determines the effective communication tool and the scale of relationship between parties. See the table 1 below that shown some examples on the parties that can set up SLA between them [9].

Table 1. Examples of SLA Parties

\begin{tabular}{|l|l|}
\hline \multicolumn{1}{|c|}{ Between } & \multicolumn{1}{c|}{ And } \\
\hline Service vendors and suppliers & Client Companies \\
\hline IT departments & Internal business units \\
\hline A help desk & Internal business units \\
\hline A marketing department & Cooperate departments \\
\hline A human recourses department & Cooperate departments \\
\hline
\end{tabular}

Manage all complaints that received from the customer to know the level of service that needed to be achieved through the mechanism that helps the manger to address all these complaints. It also assists them in preventing differences and conflicts as both the parties are aware of what expected from each other.

If there are any conflicts, the SLA helps in resolving them. This step helps the manager to addressed customer expectations based on what the customer complaints before and what they want exactly, which leads the manager classifies the solution for customers complain [7]. But there are various level of services provided depend on the customer itself and his/her nature work, also on the requested services that guided to build the matrix which described the service with its performance level [9].

The Service-Performance Matrix clarifies the service and the performance for each service, the customer request what he wants to be based on the contract that he/she signed because the level of performance effects on the money that already paid by customer. Also, the type of customer as internal or external effected on the type of requested services. The following table 2 is shown the Service-Performance Matrix [9].

Table 2. The Service-Performance Matrix

\begin{tabular}{|c|c|c|c|}
\hline & \multicolumn{3}{|c|}{ Response time } \\
\hline $\begin{array}{c}\text { Service } \\
\text { level }\end{array}$ & low & Medium & high \\
\hline S1 & $50 s$ & $25 s$ & $5 s$ \\
\hline S2 & $10 s$ & $5 s$ & $2 s$ \\
\hline S3 & $100 s$ & $50 s$ & $10 s$ \\
\hline S4 & $35 s$ & $15 s$ & $10 s$ \\
\hline
\end{tabular}

After the customer chooses the service then the manager gauges the quality of service (QoS), such as Delivery time, Performance, Availability, Time, Speed, Accuracy, Response, Security, reliability (MTBF, MTTR, Response time). The matrix is a measurement criterion that used to decide the effect in availability achieved by implementing service quality definitions. SLA helps the customer to complete a task, product a result or support third-party.

At the end of the contract the SLA manager is responsible to measure service level quality success. This can be done whether SLA are in place. It should measure the service level quality through monthly meeting with customer responsible for measuring and providing defined service levels. User groups may also present when SLA involved. The purpose of the meeting is to check performance of the measured service level definitions and to make improvements to meet customer satisfaction.

Each meeting should have a defined agenda that includes:

- Review of measured service levels for the given period.

- Review of improvement advantages defined for different customer.

- Review of current service level metrics and comparing it with customer satisfaction.

- Reporting of what improvements needed to be based on the customer satisfaction and Service Performance matrix.

- Addressing the compliance to decide the effectiveness solution based on the root problems.

All the above processes followed to make SLA successful as effective and efficient as well as within customer satisfaction.

\section{PROPOSED MODEL}

On the basis of client's requirement, the customer needs to access on database through some queries. Cloud service providers cooperate with SLA to optimize the queries into closer queries of customer's request with selected performance level. This guided us to suggest a proposed model in Figure 3 below to meet optimal results.

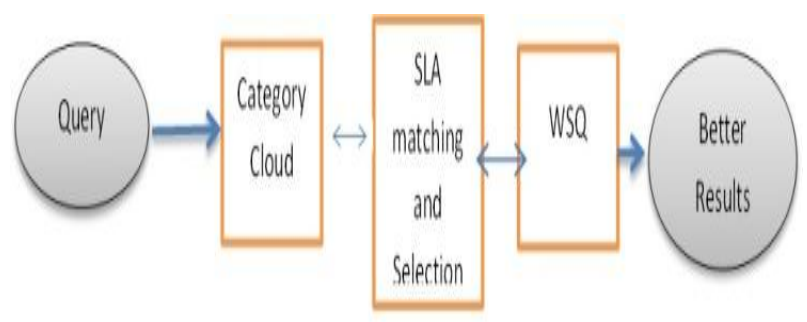

Figure 3: Proposed Model

The proposed model has sequential steps starts with query and ends with better result. The query is conditional information that requested from the customer depends exactly what he/she wants. For example select, delete or retrieve some data. Then classify the queries based on categories to simplify the search process in category cloud step. Category Cloud is classified the information as categories such as music, sports, games, foods, films etc. to simplify the search field [10].

The way to design SLAs is to supply appropriated data or metrics for a client to select services depending on the preferred one and its level of quality. Usually, SLAs are stated 
in basic content, using forms or toolkit. Providers design their systems in an approach that measurements are gathered and then matched to the metrics depending on the type of search fields determined in the SLA.

There are three major SLA categories: 1. Basic - an SLA with well-organized metrics that are calculated and/or established. The gathering of this metrics is usually completed physically. 2. Medium - a multi-stage dominance depending on the cost of the service. The goal is to equalize the stages of dominance and cost. 3. Advanced - dynamic scattering of resources to achieve requirements [11]. It is an umbrella that characterized the demand services into specific categories that are always on, anywhere, anytime and anyplace.

SLA matching and selection is considered as filter approach. It is helped to decrease the research results that matching the query that is requested by the customer [12] and also is taken the consideration for any complaints that received before from customer.

The process of matching semantically equal SLA basics from differing SLA templates is important for enabling automatic provider selection in cloud categories. This process is executed in three steps. First, for each pair of two SLA elements, the possibility for their similarity (i.e., their equivalence) is computed. Then, based on the computed probabilities, the equivalence of the elements is determined.

Finally, if two SLA elements are semantically identical, the SLA mappings are automatically established to bridge the possible differences [9]. When measuring the similarity of a pair of SLA elements, it considers their two properties: element definition, stating basic properties such as name and measurement unit, and element metric, describing a method for measuring the element value to be selected as optimal matching query [13]. This process is considered the first step of filtering procedure.

The role of WSQ (Web Supported Query) is the part generated to final filter for all the queries based on the specific category. WSQ is based on defining two virtual tables, web pages and web count to any relational database. A single query that is being executed by parallel relational query processors is allowed by Asynchronous Iteration method to hold multiple concurrent Web searches. It is responsible on all the detailed related to specific service. That leads to sort search and to match exactly the level of services that requested by internal or external customer [9].

Finally, the outcome will be as well as optimal results that lead to decrease the number of complaints from the customer and safe the customer time.

The advantage of this model is eliminating the customer problems that related to time that need to response for any request. Also, the problem of insufficient response which means provide any service that will not match exactly the services that requested by customer.

The proposed SLA model will promote and encourage the internal or external customers used within their domains.

\section{CONCLUSION}

This paper presented the new approach relating to SLA management and Cloud Computing using big data management to promote SLA application. The SLA should act as a guide for handling potential problems. It needs to look at the SLA as a tool for protecting the stability of the service, protecting the effects of the company and decreasing the expense where drastic actions are required. SLA is proper agreement between the facilitator and client to authenticate the service quality delivered in order to satisfy the needs of the query requested by clients. This also helps to maintain relationship between the both parties. Big data is applied in order to classify the web search enhancement using WSQ. Web Supported Query classifier integrated with big data to support web searches through information visualization for enhancing the search requested by the user and manages the amount of information. In future, the idea presented here will guide on linking end users expectations and using big data deploying cloud management leading as an explorer of the new way of dealing with the advance search at advance level to reach into optimal search result and decreasing the number of complaints by internal or external customer.

\section{FUTURE DIRECTION}

Service Level Agreement is vast field. There are many issues and more work required in this area. In Future more work related to SLA consumers, SLA providers with big data is on the way with new technology integration. Web based SLA is also under consideration for the new approaches for better services provided to end users. Estimate SLA proposed model pricing will be investigate in the future and enhanced network application through SLA application on it. More maturity is required in this area.

\section{REFERENCES}

[1] J. More, and C. S. Lacerda, "Service Level Agreement Business Management Strategies, July. 2016.

[2] K. Associates, "Service level agreements (SLA) author speaker," inhttp://www.servicelevelagreements.com/, 2014. [Online]. Available: http://www.servicelevelagreements.com/. Accessed: Oct. 19,2016

[3] D. Cackelt, A. Bond and J. Gouk, "Information management and Big Data, A referenced Architecture" Oracle Corporation, Feb. 2013.

[4] Andrieux, A., Czajkowski, K., Dan, A., Keahey, K., Ludwig, H., Nakata, T., Pruyne, J., Rofrano, J., Tuecke, S., \& Xu, M. (2007). Web Services Agreement Specification (WSAgreement).

[5] J. McCarthy and R. Yahyapour, Service level agreements for cloud computing: 2012, P. Wieder, J. M. Butler, and W. Theilmann, Eds. New York: Springer-Verlag New York, 2011.

[6] A.AbdusSalamQureshi and S. Mohammad Khalid Jamal, "Web supported query Taxonomy Classifier," International Journal of Computer Applications, vol. 52, no. 8, pp. 47-55, Aug. 2012.

[7] G. Blokdijk and I. Menken, The service level agreement Sla guide - Sla book, Templates for service level management and service level agreement forms. Fast and easy way to write your Sla. United States: Emereo Pty, 2008.

[8] P. C. Zikopoulos, C. Eaton, D. Dirk deRoos, T. Deutsch, and G. Lapis, Understanding Dig Data, Analytics for Enterprise Class Hadoop and Streaming Data, S. Sit, Ed. New York Chicago San Francisco: Mc Graw Hill, 2012.

[9] N. KARTEN, How to Establish Service Level Agreement. Randolph, Mass., USA: Karten Associates, 2003. 
[10] A. García García and I. Blanquer, "Cloud services representation using SLA composition,"Journal of Grid Computing, vol. 13, no. 1, pp. 35-51, Apr. 2014.

[11] C. S. Yeo et al., "Utility Computing on Global Grids," in The Handbook of Computer Networks. 2007.

[12] K. Stamou, V. Kantere, and J.-H. Morin, "SLA Template Filtering: A Faceted Approach," in The Fourth
International Conference on Cloud Computing, GRIDs, and Virtualization, Valencia, Spain, Paper 978-1-61208271-4, IARIA: CLOUD COMPUTING 2013, 2013.

[13] C. Redl, I. Breskovic, I. Brandic, and S. Dustdar, "Automatic SLA Matching and Provider Selection in Grid and Cloud Computing Markets," in IEEE 13th International Conference on Grid Computing, Beijing: IEEE, 2012, pp. 85-94. 